\title{
A Clinical, Laboratory and Microbiological Evaluation on Efficacy of Dental Air Force Home Dental Cleaning System on Type 2 Diabetic and Nondiabetic Adult Chronic Periodontitis Patients: A 6-Month Clinical Study
}

\author{
Shubangi Mani, Ameet Madan Mani, Rajiv Saini
}

\begin{abstract}
Aim: The study outlined to evaluate the clinical, laboratory and microbiological efficacy of Dental Air F orce home dental cleaning system on type 2 diabetes and nondiabetic adult chronic periodontitis patients.
\end{abstract}

Materials and methods: A total of 100 adult chronic periodontitis subjects were recruited voluntary for this study. Clinical [plaque index (PI), gingival index (GI) and clinical attachment loss (CAL)], laboratory (C-reactive protein levels and glycated hemoglobin) and microbiological parameters were measured prior to phase 1 therapy; at 3rd and 6th month post phase 1 therapy. Comparative assessment was done among all the patients that were divided into four groups with Student paired t-test and analysis of variance (ANOVA).

Results: The results of this study showed that there was significant decrease in clinical, laboratory and microbiological parameters from baseline to 6 months in all the groups (i.e. $p<0.01$ ). The subjects under groups using Dental Air Force home dental cleaning system showed highly significant reduction to all the parameters as compared to subjects under groups using toothbrush.

Conclusion: There was strong correlation between periodontal diseases and systemic parameters. Dental Air Force home dental cleaning system with the access to interdentally area helps in preventing the accumulation of dental plaque biofilm, thus able to maintain the clinical, laboratory and microbiological parameters at much lower levels as compared to traditional tooth brushing after phase I therapy.

Keywords: Dental Air Force, CR P, Diabetes, HbA1C.

How to cite this article: Mani S, Mani AM, Saini R. A Clinical, Laboratory and Microbiological Evaluation on Efficacy of Dental Air Force Home Dental Cleaning System on Type 2 Diabetic and Nondiabetic Adult Chronic Periodontitis Patients: A 6-Month Clinical Study. Int J Experiment Dent Sci 2013;2(1):1-8.

Source of support: Nil

Conflict of interest: None declared

\section{INTRODUCTION}

Periodontitis is a destructive inflammatory disease of the supporting tissues of the teeth and is caused either by specific microorganisms or by a group of specific microorganisms, resulting in progressive destruction of periodontal ligament and alveolar bone with periodontal pocket formation, gingival recession, or both. ${ }^{1}$ The predominant anaerobic microorganisms that play significant roleare Aggregatibacter actinomycetemcomitans, Porphyromonas gingivalis, Prevotella intermedia, Tannerella forsythia, $F$ usobacterium nucleatum, P eptostreptococcus micros, and C ampylobacter rectus. ${ }^{2}$ Association of periodontal infection with organ systems like cardiovascular system, endocrine system, reproductive system, and respiratory system makes periodontal infection a complex multiphase disease. Inflamed periodontal tissues produce significant amounts of proinflammatory cytokines, mainly interleukin-1 beta

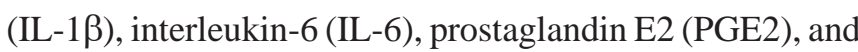
tumor necrosis factor alpha (TNF- $\alpha$ ), which may have systemic effects on the host. Periodontitis initiates systemic inflammation and can be monitored by inflammatory markers like C-reactive protein (CRP) or fibrinogen levels. ${ }^{3}$ Retention and survival of microorganisms on toothbrushes represent a possible cause of contamination of the mouth. Toothbrushes used regularly become contaminated with microorganisms, which colonize the oral cavity. I Indeed, Gerba's studies show that water droplets in an invisible cloud travel 6 to 8 feet out and up, so the areas of bathroom not directly adjunct to the toilet are still contaminated and contaminated toothbrushes may play a role in both systemic and localized diseases. ${ }^{5}$ Depending on the storage conditions, the toothbrush can, therefore, serve as a reservoir for the reintroduction of potential pathogens. ${ }^{4}$ Thus, it is apparent that present toothbrushes that were basically designed years back need to be re-evaluated. ${ }^{6}$ The new FDA-approved innovative devices like D ental A ir Force home dental cleaning system will emerge as a true alternative for conventional tooth brushing in regular practice. ${ }^{7}$ So, this study was outlined to determine the clinical [gingival index (GI), plaque index (PI) and clinical attachment loss (CAL)], laboratory (CRP and glycated hemoglobin) and microbiological (Aggregatibacter actinomycetemcomitans, F usobacterium nucleatum, Porphyromonas gingivalis and Prevotella intermedia) evaluation on efficacy of Dental A ir Force home dental cleaning system on type 2 diabetic and nondiabetic adult chronic periodontitis patients. 


\section{MATERIALS AND METHODS}

The present study was conducted in the Department of Periodontology, Pravara Institute of M edical Sciences, L oni, $M$ aharashtra, India. The research protocol was approved by the U niversity R esearch and E thical Committee. V erbal and written informed consent was obtained from all subjects prior to their voluntarily enrollment in the study.

\section{Study Population}

The subjects enrolled in this study were selected from the outpatient Department of Periodontology, Rural Dental College and Hospital, Loni, A hmednagar, M aharashtra, India. The study included a total of 100 subjects of which 50 subjects were type 2 diabetes mellitus patients with chronic periodontitis and 50 subjects were nondiabetic with chronic periodontitis. All the 100 subjects were grouped in four categories and each group was comprised of 25 subjects each as illustrated in Table 1. Exclusion criteria for the patient enrolled in the study were: (1) Presence of any systemic neurological disorder (e.g. epilepsy or schizophrenia), (2) presence of a disease with possible effects on the immune system (e.g. chronic infections or cancer), (3) patient who have received antibiotics or NSA IDS (like ibuprofen) in past 9 to 11 weeks, (4) patients who have received periodontal treatment in past 6 months, (5) pregnant and lactating mother, (6) patient with artificial prosthesis, (7) patients who smokes or consumes tobacco in any form, (8) patients suffering with arthritis, (9) patient with any type of heart disease (MI, CHD, etc), (10) female patient using intrauterine birth control devices or birth control pills, (11) obese individuals ( 30 and above range as per W HO BM I cutoff for weight categories for A sians) and (12) participants not willing to participate in the study.

\section{Clinical Protocol}

A fter the enrollment of the subjects in the study, phase 1 therapy (scaling and root planing) was done by similar EM S ultrasonic scaler by the same operator to all the 100 subjects atfirst visit/baseline only. A fter phase 1 therapy, subjects under groups B and D were advised to brush twice daily for 5 minutes by modified bass method (technique demonstrated to each subject) and similar medium bristle toothbrush and toothpaste is provided to each of the subject during the study course. A fter phase 1 therapy, subjects under groups $A$ and $C$ were advised to use D ental $A$ ir Force home dental cleaning system (technique demonstrated to each subject) twice daily for 5 minutes. Recall visits were scheduled for all the subjects belonging to all the groups ( $A$ to $D$ ) on 3rd and 6th month and no phase 1 therapy (scaling and root planing) was done at the recall visits. A II the clinical, laboratory and microbiological parameters of 100 subjects enrolled in the clinical trial were recorded at the baseline, 3rd and 6th month.

\section{Clinical Parameters Evaluated}

Clinical parameters of periodontal disease that were evaluated were GI, PI and CA L. A II the measurements were done by a single operator with W illiam's periodontal probeat base level, 3rd and 6 th month for all the groups (A to $D$ ).

\section{Laboratory Parameters Evaluated}

\section{C-reactive Protein Test}

RHELAX-CRP slidetest kit was used for the in vitro detection of $C R P$ in human serum by qual itative and quantitative rapid latex slide test. RHELAX-CRP slide test for detection of CRP is based on the principle of agglutination. The test specimen (serum) is mixed with RHELAX-CRP latex reagent and allowed to react. If CR P concentration is greater than $0.6 \mathrm{mg} /$ $\mathrm{dl}$ a visible agglutination is observed. If CRP concentration is less than $0.6 \mathrm{mg} / \mathrm{dl}$, then no agglutination is observed. No special preparation of the patient was required prior to specimen collection. $2 \mathrm{ml}$ venous blood was collected into sterile disposable tube and processed with centrifuge at 10,000 rpm for serum separation. Only serum was used for testing. If there was delay in testing occurs then samples were stored at 2 to $8^{\circ} \mathrm{C}$. A II the measurements were done at base level, 3rd and 6 th month for all the groups (A to D).

Table 1: Distribution of chronic periodontitis patients in study groups

\begin{tabular}{lll} 
Groups & Patient clinical protocol & No. of subjects \\
\hline Group A & $\begin{array}{l}\text { Type } 2 \text { diabetes mellitus patients with chronic periodontitis receiving phase I therapy followed by } \\
\text { use of Dental Air Force home dental cleaning system as regime for oral hygiene }\end{array}$ & 25 \\
Group B & $\begin{array}{l}\text { Type } 2 \text { diabetes mellitus patients with chronic periodontitis receiving phase I therapy followed by } \\
\text { conventional use of toothbrush and toothpaste as regime for oral hygiene }\end{array}$ & 25 \\
Group C & $\begin{array}{l}\text { Nondiabetic subjects with chronic periodontitis receiving phase I therapy followed by use of } \\
\text { Dental Air Force home dental cleaning system as regime for oral hygiene } \\
\text { Group D }\end{array}$ & $\begin{array}{l}\text { Nondiabetic subjects with chronic periodontitis receiving phase I therapy followed by conventional } \\
\text { use of toothbrush and toothpaste as regime for oral hygiene }\end{array}$
\end{tabular}


A Clinical, Laboratory and Microbiological Evaluation on Efficacy of Dental Air Force Home Dental Cleaning System

\section{Glycated Hemoglobin Estimation (HbA1c)}

The venous blood was subjected for assessment of $\mathrm{HbA} 1 \mathrm{C}$ test using $\mathrm{NycoC}$ ard $\mathrm{K}$ it analysis. The $\mathrm{N}$ ycoC ard $\mathrm{H}$ bA $1 \mathrm{c}$ test is a 3-minute point of care test for measurement of $\mathrm{HbA} 1 \mathrm{C}$. $\mathrm{NycoC}$ ard $\mathrm{HbA}$ 1c provides an accurate and reliable method to monitor metabolic control for diabetes mellitus. $\mathrm{NycoC}$ ard ${ }^{\circledR} \mathrm{HbA} 1 \mathrm{c}$ is a boronate affinity assay. The kit contains test devices with a porous membrane filter, test tubes prefilled with reagent and a washing solution. The reagent contains agents that lyse erythrocytes and precipitate hemoglobin specifically, as well as blue boronic acid conjugate that binds cis-diols of glycated hemoglobin. The $\mathrm{NycoC}$ ard Reader II is a small color reflectometer which can measure four different analytes by use of specific test cards, one of which is for $\mathrm{HbA} 1 \mathrm{c}$. A Il the measurements were done at base level, 3rd and 6 th month for the type 2 diabetic groups ( $A$ and $B)$.

\section{Microbiological Parameters Evaluated}

Subgingival plaque samples were collected for specific bacterial examination, i.e. Aggregatibacter actinomycetemcomitans, F usobacterium nucleatum, Porphyromonas gingivalis and P revotella intermedia. The sample sites were first wiped clean by cotton rolls to removesupragingival plaque and debris. The sample sites were then isolated using the cotton rolls and air dried. Subgingival plaque samples were then collected from the sample sites using the standardized paper point (Dentsply ${ }^{\circledR}$ ) which were inserted to the depth of the periodontal pocket until resistance was felt. The paper points were retained for 20 seconds in the collection sites. The samples site selected was maxillary first molar in all the cases to maintain the standard protocol. A fter 20 seconds the paper point was removed from the sample site and immediately transferred into the R obertson's cooked meat medium (RCM) in a test tube for specific bacterial culturing. The media was transported to the microbiological laboratory. In the laboratory the RCM was subjected to vortex homogenization for 60 seconds before incubated anaerobically (G as pack system) for 2 to 3 days. A fter 2 to 3 days incubation the growth in the RCM was subcultured on to Trypticase soy agar and B rucella agar. The bacterial growth in Trypticase soya agar and B rucella agar was assessed by semiquantitative method. All the measurements were done at base level, 3rd and 6th month for all the groups ( $A$ to $D$ ). Standard loop of $4 \mathrm{~m}$ diameter which can hold $0.01 \mathrm{~mm}$ of RCM was used and spread on to the cul ture plates, colony forming units (CFU) were counted using colony counter.

\section{Dental Air Force Home Dental Cleaning System}

Dental A ir Force is a home dental cleaning device. It is an el ectrical delivery device that uses a 1/8th $\mathrm{HP}$ oil-less electric air compressor air source with twin pistons connected to a handpiece by a pneumatic cord directed through a handpiece and tip where air at $40 \mathrm{psi}$ though a 0.020 size orifice has the introduction of a slurry of dental cleaner. This produces a jet stream of wet abrasive whereby the user directs the cleaner components and air into the sites between the teeth and below the gum line. D ental A ir Force through electron microscopy has been shown to be much less abrasive than a toothbrush and toothpaste combination. There is no electricity in the handpiece and the air source can be turned on and off remotely through a pneumatic button on the handpiece. The manufacturer recommends twice a day usage for 5 minutes as a part of the regular oral hygiene. Dental A ir Force home dental cleaning system used in this study is approved by FDA Vide No K 001493 as safety device for plaque removal in order to prevent gingivitis. The appliance uses a precision jet of air to deliver water and dental cleaner to 'pow er wash' the mouth. Dental A ir Forceuniquely dispenses the dental cleaner by you controlling the amount of cleaner that is dispensed in your mouth. One normal application uses one teaspoon of dental cleaner. The cl eaner ingredients include sodium bicarbonate, the most widely accepted and totally natural buffering agent that promotes a neutral environment. It also contains mint flavoring, $X$ ylitol and Stevia as natural sweeteners. Theformula is free of sodium lauryl sulfate, the ingredient in most toothpaste that causes sensitivity and irritation. ${ }^{8}$

\section{RESULTS}

Distribution of mean and standard deviation values of all the clinical, laboratory and microbiological parameters of all the groups (A to D) were illustrated in Tables 2 and 3. After applying Student's paired t-test there was a highly significant decrease in clinical, laboratory and microbiological parameters from baseline to 6 months in groups A, B, C and D (i.e. $p<0.01$ ). It was observed that group $C$ showed more significant decrease as compared to group A (i.e. $p<0.01$ ). A lso by applying Student' s unpaired t-test there was a highly significant difference between mean values of all clinical, laboratory and microbiological parameters in group A vs $B$, group $A$ vs $C$, group $A$ vs $D$, group $B$ vs $C$, group $B$ vs $D$, and group $C$ vs $D$ (i.e. $p<0.01$ ). It was concluded that the mean clinical, laboratory and microbiological parameters in group $C$ showed larger decrease than group $A(p<0.01)$ as shown in Graph 1 (A vs B), Graph 2 (C vs D) and Graph 3 (A vs C). By applying two-way ANOVA (Tukey-K ramer multiple 
Table 2: Distribution of mean and standard deviation values (mean $\pm S D$ ) of all clinical and laboratory parameters at baseline, 3 rd and 6th months in all the experimental groups

\begin{tabular}{|c|c|c|c|c|c|c|}
\hline Groups & Observation & GI & $\mathrm{PI}$ & $C A L$ & CRP & $\mathrm{HbAlc}$ \\
\hline \multirow[t]{3}{*}{ Group A } & Baseline & $2.62 \pm 0.25$ & $2.56 \pm 0.24$ & $5.91 \pm 0.32$ & $2.5 \pm 0.59$ & $8.62 \pm 1.08$ \\
\hline & 3rd month & $1.0 \pm 1.88$ & $1.51 \pm 0.12$ & $3.71 \pm 0.74$ & $1.42 \pm 0.73$ & $7.96 \pm 1.12$ \\
\hline & 6 th month & $0.70 \pm 0.21$ & $1.0 \pm 0.28$ & $2.40 \pm 0.61$ & $0.94 \pm 0.75$ & $7.58 \pm 1.17$ \\
\hline \multirow[t]{3}{*}{ Group B } & Baseline & $2.95 \pm 0.23$ & $2.65 \pm 0.26$ & $5.69 \pm 0.92$ & $3.12 \pm 0.48$ & $8.40 \pm 0.79$ \\
\hline & 3rd month & $1.18 \pm 0.19$ & $1.34 \pm 0.20$ & $4.23 \pm 0.72$ & $2.28 \pm 0.77$ & $8.10 \pm 0.81$ \\
\hline & 6 th month & $1.21 \pm 0.23$ & $1.27 \pm 0.27$ & $3.38 \pm 0.49$ & $2.16 \pm 1.01$ & $7.93 \pm 0.81$ \\
\hline \multirow[t]{3}{*}{ Group C } & Baseline & $2.53 \pm 0.31$ & $2.48 \pm 0.24$ & $5.88 \pm 3.69$ & $2.78 \pm 0.57$ & $5.56 \pm 0.51$ \\
\hline & 3rd month & $1.55 \pm 0.25$ & $1.1 \pm 0.11$ & $3.69 \pm 0.55$ & $1.70 \pm 0.70$ & $5.34 \pm 0.51$ \\
\hline & 6th month & $0.82 \pm 0.37$ & $0.97 \pm 0.06$ & $2.52 \pm 0.50$ & $1.22 \pm 0.74$ & $5.26 \pm 0.52$ \\
\hline \multirow[t]{3}{*}{ Group D } & Baseline & $2.64 \pm 0.28$ & $2.6 \pm 0.26$ & $5.8 \pm 0.25$ & $2.95 \pm 0.42$ & $5.48 \pm 0.52$ \\
\hline & 3rd month & $1.41 \pm 0.16$ & $1.19 \pm 0.21$ & $4.09 \pm 0.51$ & $2.11 \pm 0.52$ & $5.36 \pm 0.52$ \\
\hline & 6th month & $1.42 \pm 0.14$ & $1.21 \pm 0.19$ & $3.29 \pm 0.43$ & $1.99 \pm 0.87$ & $5.32 \pm 0.51$ \\
\hline
\end{tabular}

GI: Gingival index; PI: Plaque index; CAL: C linical attachment loss; CRP : C-reactive protein; HbAlc: Glycated hemoglobin

Table 3: Distribution of mean and standard deviation values (mean $\pm S D$ ) of all microbiological parameters at baseline, $3 \mathrm{rd}$ and 6 th months in all the experimental groups

\begin{tabular}{llcrrr} 
Groups & Observation & \multicolumn{1}{c}{$\mathrm{Aa}$} & $\mathrm{Fn}$ & $\mathrm{Pg}$ & $\mathrm{Pi}$ \\
Group A & Baseline & $26.72 \pm 4.47$ & $31.16 \pm 5.71$ & $26.36 \pm 5.31$ & $24.8 \pm 6.34$ \\
& 3rd month & $13.48 \pm 3.55$ & $16.96 \pm 2.26$ & $13.04 \pm 3.51$ & $12.52 \pm 3.74$ \\
Group B & 6th month & $4.64 \pm 1.7$ & $5.0 \pm 1.76$ & $4.40 \pm 2.12$ & $5.24 \pm 2.63$ \\
& Baseline & $31.12 \pm 7.5$ & $32.32 \pm 5.81$ & $30.4 \pm 6.09$ & $31.72 \pm 6.19$ \\
& 3rd month & $26.84 \pm 7.23$ & $29.52 \pm 5.91$ & $27.04 \pm 5.26$ & $28.52 \pm 5.93$ \\
Group C & 6th month & $24.08 \pm 7.32$ & $27.04 \pm 5.83$ & $24.72 \pm 4.95$ & $25.96 \pm 5.79$ \\
& Baseline & $28.24 \pm 3.5$ & $29.72 \pm 3.71$ & $28.04 \pm 3.07$ & $28.4 \pm 3.45$ \\
Group D & 3rd month & $17.32 \pm 4.19$ & $18.24 \pm 5.25$ & $13.68 \pm 3.69$ & $16.4 \pm 5.47$ \\
& 6th month & $7.32 \pm 4.25$ & $6.08 \pm 1.61$ & $5.76 \pm 2.0$ & $6.48 \pm 2.81$ \\
& Baseline & $25.88 \pm 4.62$ & $28.4 \pm 6.49$ & $26.92 \pm 5.14$ & $28.76 \pm 4.64$ \\
& 3rd month & $23.36 \pm 4.37$ & $26.32 \pm 6.42$ & $24.28 \pm 4.73$ & $26.04 \pm 4.38$
\end{tabular}

Aa: Aggregatibacter actinomycetemcomitans, Fn: Fusobacterium nucleatum, Pg: Porphyromonas gingivalis, P i: Prevotella intermedia

comparison test) test there is a significant difference between groups A , B , C and D when compared together in respect to clinical, laboratory and microbiological parameters $(p<0.05)$.

\section{DISCUSSION}

The oral cavity is a portal of entry as well as the site of disease for microbial infections that affect general health. Periodontitis has been proposed as having an etiological or modulating role in cardiovascular disease, diabetes, respiratory disease and adverse pregnancy outcome and several mechanisms have been proposed to explain or support such theories and oral lesions are indicators of disease progression and oral cavity can be a window to overall health and body systems. ${ }^{9}$ Locally produced inflammatory mediators such as CRP, interleukins- 1 beta (IL-1 $\beta$ ), interleukins 6 (IL-6), tumor necrosis factor al pha (TNF- $\alpha$ ) are the key modulating agents in cardiovascular disease. Periodontitis creates a burden low level of systemic inflammatory reactants (CRP) bacterial pathogens, antigens, endotoxins and inflammatory cytokines (IL-1, IL-6, TNF- $\alpha$ ) that contribute to the process of atherogenesis and thromboembolic events. ${ }^{10}$ The key for optimal oral health that lead to better systemic heal th is achieved by regular daily oral hygiene, Dental A ir Force home dental cleaning system showed significant results in achieving the same as compared to conventional toothbrush.

The subjects (groups A and C) using Dental A ir Force home dental cleaning system showed more significant clinical, laboratory and microbiological results in both type 2 diabetic and nondiabetic adult chronic periodontitis patients as compared to subjects (groups B and D) using toothbrush and toothpaste after phase 1 therapy (scaling and root planing). The possible mechanism of greater efficiency as compared to toothbrush is that Dental A ir Force home dental cleaning system uses air and a dental cleaner with water to break through the plaque barrier. The air oxygenates the spaces between teeth and along the gum line, making it difficult for the anaerobic plaque-causing bacteria to live. Sodium bicarbonate is a neutralizing agent that acts on the acids produced by the bacteria. It is an abrasive that breaks up 
A Clinical, Laboratory and Microbiological Evaluation on Efficacy of Dental Air Force Home Dental Cleaning System
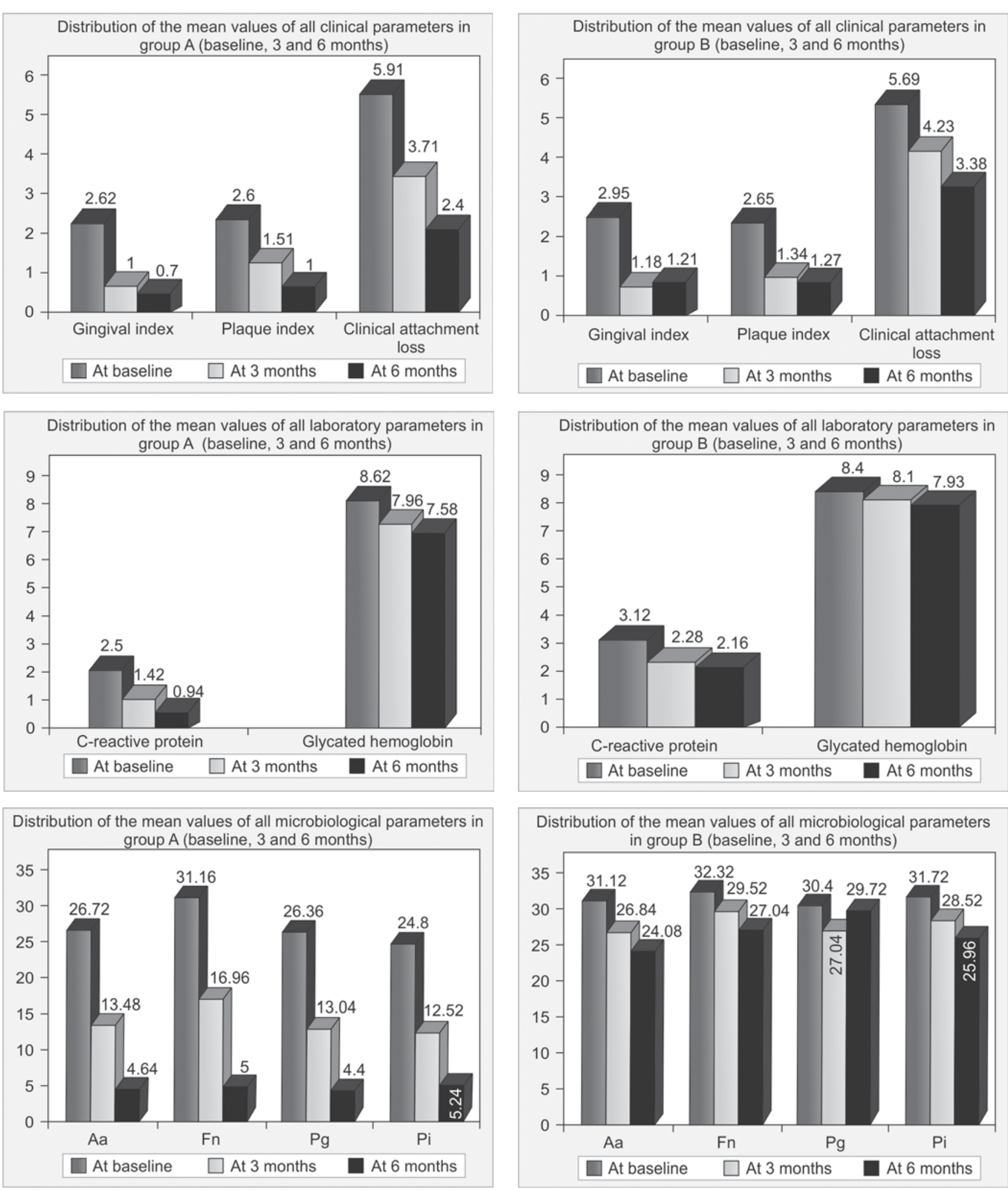

Graph 1: Comparison of groups A vs B

the plaque's sticky film. It also removes the odor caused by the plaque. The water flushes away the bacteria and debris offs the surfaces of the teeth. ${ }^{8}$ Dental Air Force home dental cleaning system with access to subgingival area lead to removal of biofilm and prevent further proliferation of periodontopathic microorganisms.

\section{CONCLUSION}

The results of this study showed that there was positive correlation with periodontitis and systemic heal th observed by elevated levels of CRP and $\mathrm{H} b \mathrm{bA}$ 1c levels. F urther this study it was established that with regular use of Dental $A$ ir Force home dental cleaning system as oral hygiene regime therewas 

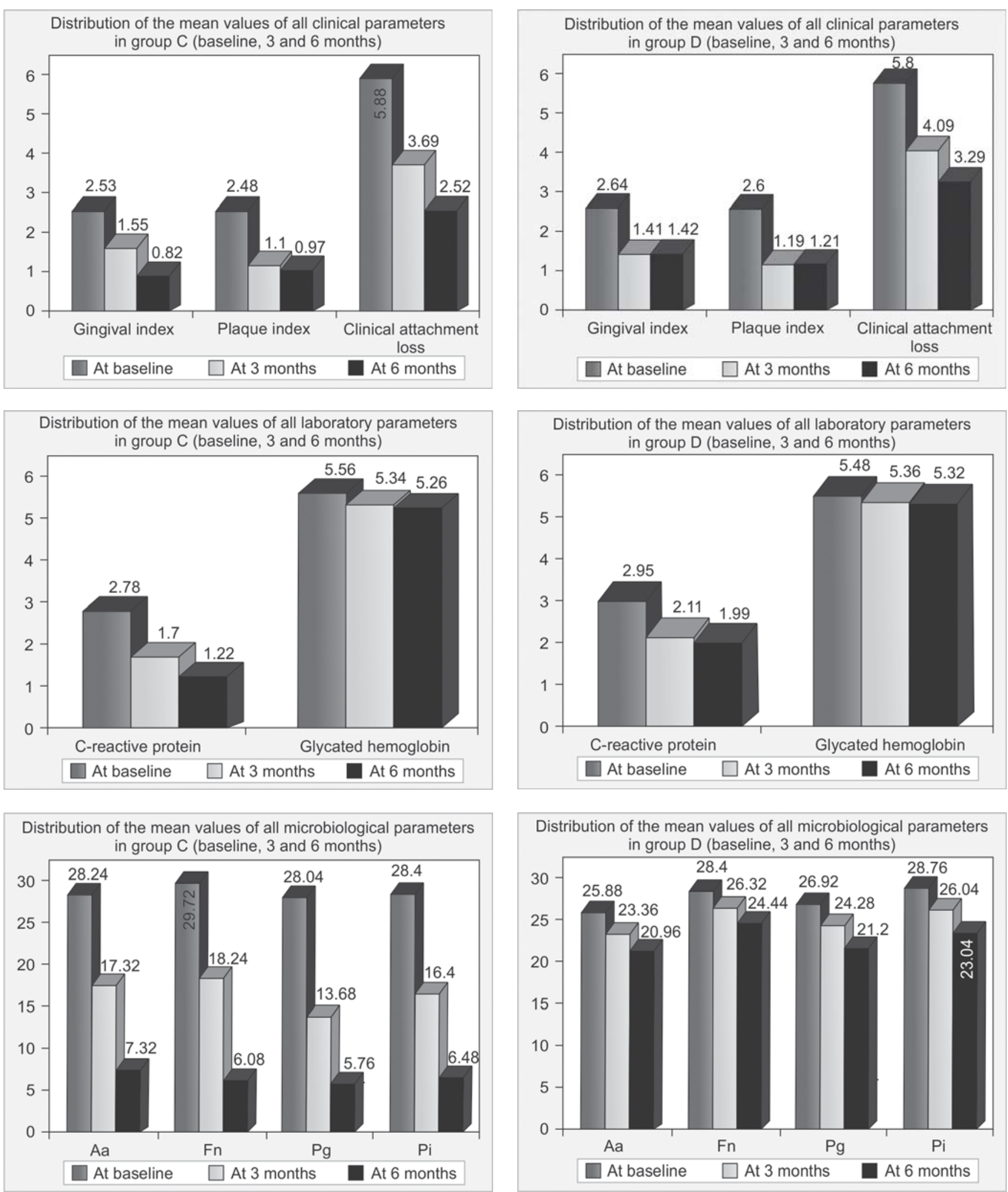

Graph 2: Comparison of groups C vs D 
A Clinical, Laboratory and Microbiological Evaluation on Efficacy of Dental Air Force Home Dental Cleaning System
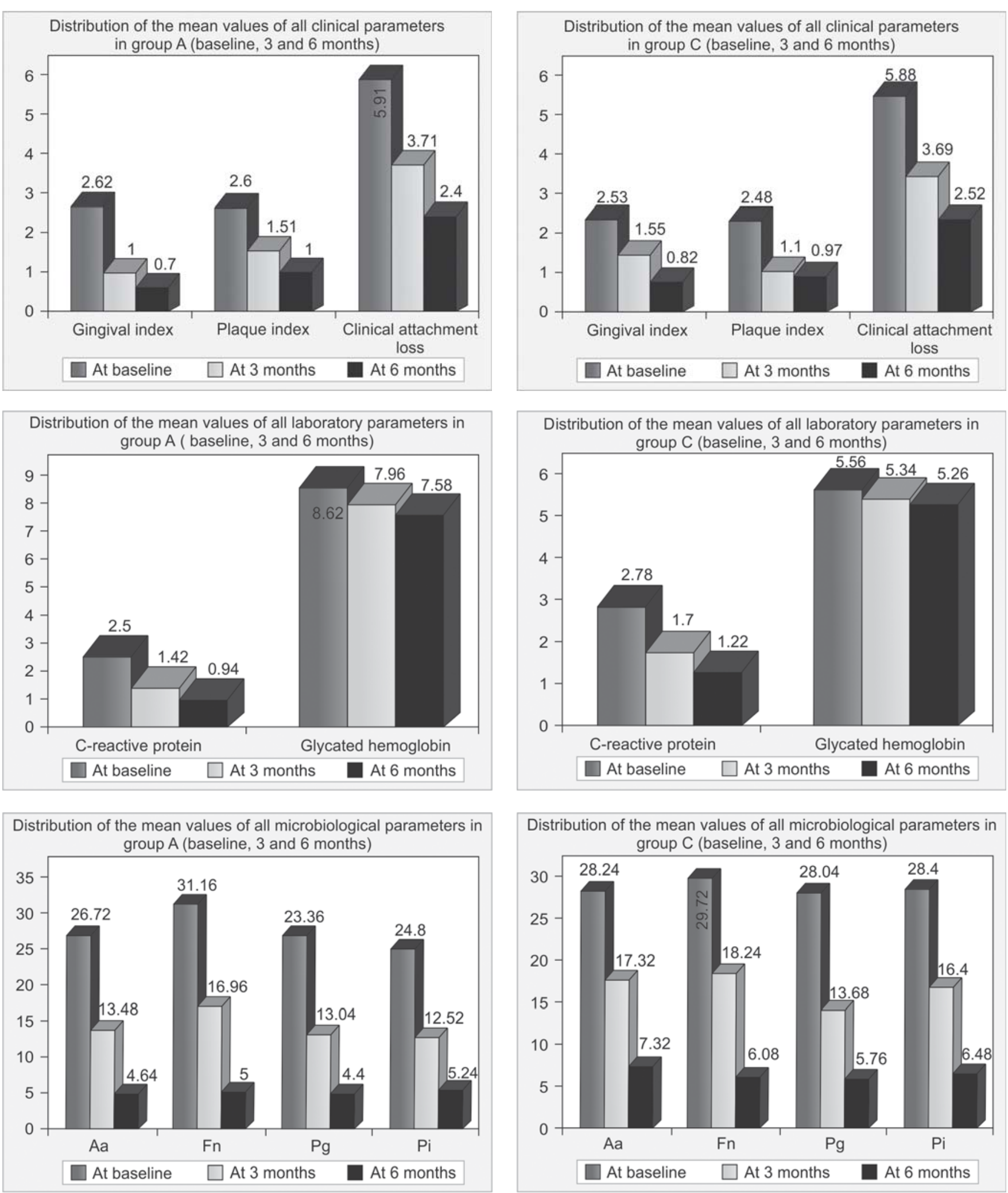

Graph 3: Comparison of groups A vs C 
significant reduction in all the clinical, laboratory and microbiological parameters of all the adult chronic periodontitis subjects as compared to toothbrush.

\section{REFERENCES}

1. Saini R, Saini S, Sharma S. Periodontal disease linked to cardiovascular disease. J Cardiovasc Dis Res 2010;1:161-62.

2. Saini R, Saini $S$, Sharma $S$. Therapeutics of stem cells in periodontal regeneration. J Nat Sc Biol Med 2011; 2:38-42.

3. Saini R, Saini S, Saini SR. Periodontitis: A risk for delivery of premature labor and low birth weight infants. J N at Sc Biol M ed 2011; 2:50-52.

4. Taji SS, Rogers AH. The microbial contamination of toothbrushes: A pilot study. A ust Dent J 1998; 43:128-30.

5. Glass RT, Lare M M. Toothbrush contamination: A potential heal th risk. Quintessence Int 1986;17:39-42.

6. Saini R, Saini S. M icroflora on toothbrush: A t greater risk. A nn Nigerian M ed 2010; 4:31-32.

7. Saini R. Periodontal health 2020: The future outl ook. Editorial. Int J Experiment Dent Sci 2012;1(2).

8. M ani A, V advadgi V, A narthe R, Saini R, M ani S. A clinical study on Dental A ir Force home dental cleaning system on adult chronic periodontitis patients and its assessment to $C$-reactive protein levels. IntJ Experiment Dent Sci 2012;1:14-18.

9. Saini R, Saini S, Saini SR. Periodontal diseases: A risk factor to cardiovascular disease. A nn Card A naesth 2010;13:159-61.

10. Piero P, Rajiv S. Hospital infection control: Clinical guidelines (1st ed). In: Saini S, Saini R (Eds). Paras Medical Publisher, Hyderabad 2012:188-96.

\section{ABOUTTHE AUTHORS}

\section{Shubangi Mani (Corresponding Author)}

A ssociate Professor, Department of Orthodontics, R ural Dental College Loni, M aharashtra, India, Phone: +91-9923206789, e-mail: drperiodontist@yahoo.co.in

\section{Ameet Madan Mani}

A ssociate Professor, Department of Periodontology, Pravara I nstitute of M edical Sciences, A hmednagar, M aharashtra, India

\section{Rajiv Saini}

A ssistant Professor, D epartment of Periodontology, Pravara I nstitute of M edical Sciences, A hmednagar, M aharashtra, India 